\title{
Juana de Ibarbourou oder die Juana Amerikas
}

Wenden wir uns nun dieser heute ebenfalls ins zweite Glied gerückten Dichterin Juana de Ibarbourou zu, die 1929 zur „Juana de América“ ausgerufen wurde und zumindest bis zur Mitte des 20. Jahrhunderts zu den großen, überragenden Stimmen der lateinamerikanischen Lyrik zählte. Die Dichterin aus Uruguay stieß auf eine kontinentale und noch in Europa spürbare Rezeption, wie sie neben Gabriela Mistral nach ihr - soweit ich sehe - keiner der Lyrikerinnen Lateinamerikas mehr zuteilwurde.

Es fällt schwer, von Juana de Ibarbourou zu sprechen, ohne ihren Habitus mitzudenken und in die Analyse miteinzubeziehen. Denn die Uruguayerin wirkte auch durch ihr mondänes Auftreten, mit dem sie sich ebenso von der Schlichtheit Gabriela Mistrals wie der feministischen Kampfeslust und tiefen Verzweiflung Alfonsina Stornis abhob, ja gänzlich unterschied. Eine uruguayische Essayistin und fanatische Verteidigerin ihrer Landsfrau betonte immer wieder Juanas Schönheit und Anmut. Auch schreckte sie nicht davor zurück, dem historischen Zusammentreffen von letzterer, Gabriela Mistral und Alfonsina Storni, das vielfach medial festgehalten wurde, sowohl die Züge dreier Grazien zu vermitteln, aber zugleich die Grazie der beiden anderen Frauen abzuwerten, indem sie Gabriela als zu „varonil“, als allzu männlich, abwertete und Alfonsina als eine „fea dulzura“, eine hässliche Süße, porträtierte. Mit der Lyrik oder ästhetischen Wirkung der Dichtungen dieser Lyrikerinnen hat das nicht das Geringste zu tun. Gerade bei den Frauen spielten der öffentliche Auftritt und die verschiedenartigen Ausdrucksformen des Habitus eine noch größere Rolle als bei den männlichen Kollegen. Wir sollten es Juana de Ibarbourou freilich nicht übel auslegen, dass sie als schöne junge Frau dem Zeitgeschmack des lesenden Bürgertums wohl besser entsprach als die beiden anderen Dichterinnen. Was für uns im Rahmen dieser Vorlesung zählt, ist die poetische Qualität der jeweiligen Dichtkunst.

Es dürfte wohl kaum eine Lyrikerin des amerikanischen Kontinents in diesem 20. Jahrhundert gegeben haben, in deren Erfolg - wenn auch nicht in die unbestrittene literarische Qualität ihrer Dichtung - sich in so penetrantem Maße der wohl erstmals massenmedial verbreitete Anblick der schönen, eleganten, attraktiven, unschuldigen Lyrikerin mischte wie bei Juana de Ibarbourou. Ich möchte diesen äußerlichen Aspekt, der heutzutage bei einer Schriftstellerin leider eine noch wesentlich größere Rolle spielt, gerne kommunikationstheoretisch verstanden wissen und damit keineswegs die literarische Qualität der hübschen Uruguayerin schmälern. Schönheit soll aber auch nicht als Handicap verstanden werden. Beschäftigen wir uns vor einem textanalytischen Durchgang noch kurz mit einigen Biographemen von Juana de Ibarbourou! 
Sie wurde am 8. März 1892 im uruguayischen Melo geboren und starb am 15. Juli 1979 in Montevideo. Sie entstammte einer wohlhabenden Familie, die mütterlicherseits zur uruguayischen Oligarchie zählte, während ihr Vater aus Galicien stammte. Auch bei ihr haben wir es mit einem ,nom de plume“ zu tun: Ihr eigentlicher Taufname lautete schlicht Juana Fernández Morales. Sie besuchte zunächst eine Klosterschule, dann ein staatliches Gymnasium und erhielt so eine fundierte literarische Bildung. 1914 heiratete sie den Hauptmann Lucas Ibarbourou und bekam 1917 ihr einziges Kind mit Namen Julio César. Ein Jahr später ließ sie sich in Montevideo nieder, in der Hauptstadt Uruguays, die sie seither kaum noch verließ.

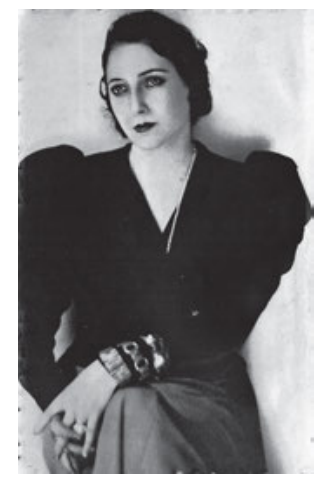

Abb. 88: Juana de Ibarbourou (Melo, Departamento Cerro Largo, Uruguay, 1895 - Montevideo, 1979).

Ihre beiden ersten, öffentliches Aufsehen erregenden Gedichtbände veröffentlichte sie in den Jahren 1919 und 1920. Beide Bände erreichten auf Anhieb ein nationales, rasch aber auch internationales Publikum. Für ihr lyrisches Werk, das im Verlauf ihres weiteren Lebens auf mehr als dreißig Bände anschwoll, erhielt sie 1929 im Palacio Legislativo von Montevideo, unter Vorsitz von Juan Zorrilla de San Martín und keinem Geringeren als Alfonso Reyes, den Ehrentitel ,Juana de América“. Mehrfach war sie als Kandidatin für den Literaturnobelpreis nominiert, von vielen lateinamerikanischen Staaten wurden ihr Ehrungen zuteil und - wie etwa 1946 von der Regierung Belgiens - höchste Auszeichnungen zugesprochen. Bereits 1941 wurde sie zur Vorsitzenden des Pen Club Uruguay und 1953 von einer Vereinigung US-amerikanischer Frauen in New York zur „Frau der Amerikas“ gewählt. Die Ehrungen sind Legion. Man darf ohne Übertreibung sagen, dass sie als Lyrikerin eine brillante Karriere genoss.

Im Jahr 1909 veröffentlichte sie ihr erstes Prosastück mit dem Titel Derechos femeninos, mit dem sie sich für die Rechte der Frauen einsetzte und - wenn wir den Begriff des Feminismus weit auslegen - feministische Positionen bezog. Mit Begeisterung wurde ihr erster Gedichtband Las lenguas de diamante von 1919 auf- 
genommen, der manchen Kritikern bis heute als ihr bestes Werk gilt. Es ist eine Dichtkunst, die großen Wert auf Schlichtheit und Präzision legt, dabei aber deutlich vom hispanoamerikanischen Modernismo geprägt ist. Anders als bei Gabriela Mistral entfaltet sich bei Juana aber das Bild einer sinnlichen Frau, was sicherlich zu ihrem Markenzeichen wird und die öffentliche Wahrnehmung ihres Habitus prägte. Die in ihren Gedichten zum Ausdruck kommende Erotik ist anders als bei ihren argentinischen Zeitgenossinnen Delmira Agustini oder Alfonsina Storni nicht herausfordernd und offensiv, sondern unbeschwert und sinnenfroh. Die flüchtige Begegnung, die kurze Berührung, der ephemere Augenblick werden zu künstlerischen Ausdrucksformen einer Sinnlichkeit und Erotik, die zumindest in ihren jungen Jahren noch nichts vom Tod wissen will. In ihrem Spätwerk, etwa in Perdida von 1950 oder Azor von 1953, machen sich auf der Ebene ihrer Bildersprache mehr und mehr surrealistische Einflüsse bemerkbar. Die Ehrungen für ihr poetisches Werk enden erst mit dem Tod der Dichterin Ende der siebziger Jahre.

Ich habe eben bemerkt, dass sich Juana de Ibarbourou sehr früh schon für die Rechte von Frauen eingesetzt hatte. Auf Grund ihrer zahlreichen Ehrungen und ihres Auftretens machte sich aber bald Protest gegen sie breit, der bis heute nicht ganz verstummt ist. Gerade in neueren Untersuchungen wie etwa von Eyda Machín ist der wenig feministische Grundton ihrer Lyrik gerügt und sie selbst häufig gegenüber Alfonsina Storni, mit der wir uns im Anschluss ebenfalls noch intensiv beschäftigen werden, abgewertet worden. ${ }^{1}$ Doch mir scheint es viel wichtiger, die uruguayische Lyrikerin zum einen an ihren eigenen Vorgaben und Ausdrucksformen zu messen und zum anderen jeglichen Wettbewerb zwischen diesen „Poetisas“ zu vermeiden, der mit wechselnden Kriterien - Schönheit, Feminismus oder Experimentalität - immer wieder zwischen Gabriela Mistral, Juana de Ibarbourou, Alfonsina Storni oder Delmira Agustini oft auch unter nationalen Vorzeichen durchgeführt wurde.

Wenig Feministisches findet sich sicherlich in dem Gedicht Soneto a Dios, das 1967 in ihrem Gedichtband La Pasajera erschien und von Eyda Machín nur mit einer kurzen, abfälligen Floskel bedacht wurde. Wir wollen es uns etwas näher anschauen, zumal es sich um ein Gedicht aus einer völlig anderen, aber uns bereits sehr nahen Zeit handelt. Und doch ist das Gedicht der damals zweiundsiebzigjährigen Juana de Ibarbourou eher der Ästhetik ihrer frühen Jahre verpflichtet. Nähern wir uns diesem Sonett der uruguayischen Dichterin ohne Vorurteile und in einer Übersetzung an, in welcher ich versucht habe, mich Rhythmus und Reimstruktur des spanischsprachigen Originals anzunähern:

1 Vgl. Machin, Eyda: La mujer y la escritura: Juana de Ibarbourou y Alfonsina Storni. In: Heydenreich, Titus (Hg.): Der Umgang mit dem Fremden. München: Fink 1986, S. 65-90. 
Weil Du mir's Wort gabst und sie

In mir sein konnte, dem Universum treu

In der geläufigen Gemme mein Vers ohne Scheu

Sich später einen leuchtenden Schild auslieh.

Als Deine Schuldnerin fühl ich mich, eile zu Dir

Bei Nacht und Tag in hell strahlendem Glanz,

Oh glückliche Stunde, der dunklen Zeit ganz

Entgegen, treue Lilie, nackte Pappel mir.

So verbeug' ich mich wie einst Hiob, geduldig,

Untertänig wartend als Büßer huldig,

In Deinem Schatten, dem Blitze geheuer,

Dein Diamant war ich, mit unschuldigem Feuer,

Und bin doch dunkle Seele, Deinem Erbarmen offeriert,

Was immer die bleiche Aurora des Maitags gebiert. ${ }^{2}$

In diesem Sonett aus dem Spätwerk Juana de Ibarbourous fallen zunächst die traditionellen Bezüge auf allen Ebenen ins Auge. Dies beginnt zum einen mit dem Titel, der in gewisser Weise doppelt eingelöst wird. Erstens handelt es sich in der Tat um ein Sonett an die Adresse Gottes, der bereits im ersten Vers aus einer Haltung der Demut, ja der Unterwürfigkeit heraus angesprochen wird. Das Gedicht hält also, was sein Titel verspricht, und wendet sich in der Form des klassischen Sonetts an Gott den Schöpfer, der dem lyrischen Ich alles gegeben oder besser alles geliehen habe. Zweitens löst das Gedicht seinen Titel auf einer anderen Ebene ein, nämlich auf der des Lebewohls, des Abschieds, des „Adios“. Es ist zugleich auch ein Abschiedsgedicht, ein Gedicht, das die Haltung des Rückblicks einnimmt und den zurückliegenden Weg aus der Perspektive einer heiteren Erfahrung dankbar reflektiert. Die Möglichkeit, im hellen Lichte zu erstrahlen und für die Menschen sichtbar zu sein, wird ganz der Allmacht Gottes zugeschrieben. Das lyrische Ich kam aus der Dunkelheit und wird als dunkle Seele wieder in diese Dunkelheit zurückkehren, ganz dem Erbarmen Gottes preisgegeben.

Es ist ein Gedicht der Dankbarkeit gegenüber dem Schöpfer-Gott, dessen Schöpfung ebenso das lyrische Ich wie das Ich der Lyrikerin ist. Zugleich ist es ein Gedicht des Abschieds, in welchem noch einmal das bisherige lyrische Werk Juana de Ibarbourous ab Las lenguas de diamante auftaucht und reflektiert wird. Damit

2 Ibarbourou, Juana de: Soneto a Dios. In (dies.): Obras completas. Buenos Aires: Aguilar 1968, S. 554 . 
erscheint die eigene lyrische Schöpfung gleichsam als Schöpfung zweiten Grades, als sekundäre Kreation, welche letztlich einem anderen, wahren „creator“ überantwortet wird. Das Sonett der Dankbarkeit ist ein in Gott ruhendes und zugleich ein in sich ruhendes Gedicht, das vor allem Reflex und Reflexion ist von einem erreichten, gewonnenen und mehr noch geschenkten, aber doch nur geliehenen Standpunkt: Stets war alles nur in Gott verankert. Zu dieser Dimension der Ruhe passt auch die materielle Struktur des Gedichts, das zweifellos nur noch wenig mit den mondänen Auftritten der Ibarbourou gemein hat, sondern uns eine im Alter tief religiöse Dichterin zeigt, welche ihre Erfolge und Auszeichnungen allein Gott verdankt.

Bereits die graphische Anordnung des Gedichts lässt deutlich erkennen, dass es sich um ein Sonett handelt, das aus insgesamt vierzehn Versen aufgeteilt in zwei Quartette und zwei Terzette besteht. Die Elfsilber sind hierbei vom Reimschema her recht einfach aufgebaut, haben wir es doch in den beiden ersten Strophen mit zweimal $a b b a$, also einem umschlingenden Reim, zu tun. Bei einem Sonett wird es ja immer, wie Sie wissen, in den Terzetten spannend, und zwar gleich in mehrfacher Hinsicht: zum einen ganz klassisch auf der Ebene des Inhalts, zum anderen auf jener der Form. Da beide Ebenen sich in einem Gedicht nur selten ,auseinanderklamüsern' lassen und vielmehr eng miteinander verwoben sind, können wir dies im konstruktiven Zusammenhang des Gedichts untersuchen.

Die generelle Struktur eines klassischen Sonetts legt nahe, dass in den beiden Quartetten die Exposition, in den nachfolgenden Terzetten die Durchführung und bisweilen die originelle Volte oder überraschende Wendung angelegt sind. Blicken wir auf den Aspekt des Reimschemas, dann wird selbstverständlich klar, dass die zueinander gehörenden Reimpaare innerhalb von Dreiergruppen, wie es Terzette nun einmal darstellen, nicht einfach angeordnet werden können. Sie müssen sich in einer sehr unterschiedlichen Weise auf die Terzette verteilen und bilden notwendige Verbindungen zwischen diesen beiden letzten Strophen. Dafür gibt es verschiedene Möglichkeiten, die ich in diesem Kontext nicht allesamt aufzählen kann, die aber in diesem Gedicht in einer sehr klaren Variante vorgeführt werden und kurz untersucht werden sollen. Dabei geht es nicht darum, mit Hugo Friedrich die Struktur der modernen Lyrik ${ }^{3}$ zu untersuchen und etwa die unermüdliche Arbeit am Sonett ausgehend von Charles Baudelaire über Paul Verlaine und Arthur Rimbaud bis ins 20. Jahrhundert nachzuzeichnen, sondern jene spezielle Form herauszuarbeiten, die Juana de Ibarbourou als die für dieses Danksagungs- und Abschiedsgedicht adäquateste erschien.

3 Vgl. die klassische Studie von Friedrich, Hugo: Die Struktur der modernen Lyrik. Von Baudelaire bis zur Gegenwart. Hamburg: Rowohlt 1956. 
Untersuchen wir also die letzten sechs Verse der Terzette dieses Soneto a Dios, so fällt auf, dass die beiden ersten Verse des ersten Terzetts in einem Paarreim miteinander verbunden sind, während die restlichen vier Verse - und damit über die Terzett- oder Strophengrenze hinausgehend - miteinander wie die Quartette zu einem Umschlingenden Reim verbunden werden. In gewisser Weise kehrt das Gedicht in die Ausgangsform der Quartette zurück, eine Tatsache, die den stark in sich ruhenden Charakter des Sonetts auf Ebene der Strophenstruktur bestätigt. Bemerkenswert ist hierbei, dass der Paarreim jeweils in Reimendstellung die beiden Lexeme „paciente“ und „penitente“ hat, so dass mithin die Geduld wie auch die Büßerschaft einen zentralen Aspekt dieses Gedichts bereits durch diese hervorgehobene Versendstellung zu betonen scheinen. Die zentrale Geste des lyrischen Ichs, die Verbeugung vor dem Schöpfer-Gott, wird just in diesem Vers vorgenommen und zugleich auch ein Verweis auf die biblische Figur des Hiob aufgenommen. In ihr spiegelt sich traditionsgemäß die Frage der Gottesgerechtigkeit, die Frage des Leidens des Gerechten und auch die Figur des Hiob als klassische Figur des Dulders und Erdulders im Rahmen der alles umspannenden Heilsgeschichte.

Die biblischen Reminiszenzen enden hier noch lange nicht, sondern bilden eine das gesamte Sonett durchziehende Isotopie. Sie beginnen im Grunde bereits im ersten Vers: Denn im Anfang war das Wort, „la palabra“, und das Wort war bei Gott und konnte darauf beim Menschen und folglich in ihr sein, als ein dem lyrischen Ich geliehenes Wort. Der Empfang des Wortes aus Gottes Hand ermöglicht überhaupt erst die weitere abgeleitete, sekundäre schöpferische Tätigkeit des lyrischen Ich, das sich in seiner gottesfürchtigen Demut ganz auf diese Zweitrangigkeit zurückzieht und die eigenen Erfolge im Bereich der Literatur minimiert. Am Ende ihres eigenen Weges bekennt sich das lyrische Ich zu dieser Rolle als Werkzeug in den Händen Gottes: „Fui tu diamante de inocente fuego.“

Die wortwörtliche Anspielung ist überdeutlich und sofort autobiographisch lesbar: Denn Juana de Ibarbourou war nichts anderes als eben jener Diamant, der in den hellen Strahlen ihres ersten Gedichtbandes Las lenguas de diamante am Horizont der Literaturen des Kontinents auftauchte und in vollem Glanz erstrahlte. Dieser Diamant, von unschuldigem Feuer erstrahlend, ist nun jedoch erloschen und in eine „alma oscura“, eine dunkle Seele, zurückgefallen, welche sich bußfertig und willig ihrem Schöpfer übergibt und anheimstellt. Was einst am Horizont der Literaturen Lateinamerikas so hell und ausgezeichnet strahlte, war nur geliehener Glanz, war nur das, was diese dunkle Seele in der Schale eines Gedichts hatte modellieren dürfen, ganz von ihrem Schöpfer-Gott geduldet.

Es fällt nicht schwer, diese Metaphorik und Gestik in den Zusammenhang des kometenhaften Aufstiegs der Lyrikerin und einer nachfolgenden langen Zeit der Dunkelheit zu beziehen, welche ihren großen Erfolgen auf einer Weltbühne folgte, 
die sie seit langen Jahren bereits wieder verlassen hatte. Die Edelstein-Metaphorik oder - wie wir etwas anzüglich auch sagen könnten - die Juweliers-Metaphorik leitet so über von der „menuda gema“ über den „reluciente escudo“ bis hin zum ehemaligen Diamanten, der wieder in eine Seele übergegangen ist, die sich in die Dunkelheit der letzten Jahre in ihrem Haus in Montevideo zurückzieht. Eine ganze Karriere wird sichtbar und verschwindet wieder in der Wortlosigkeit, in der Anonymität - für die das vorliegende Gedicht freilich der eklatante Gegenbeweis ist.

Die Entwicklung und Entfaltung des Gedichts über die im zweiten Quartett auftauchende biologische Metaphorik hinaus lässt sich sehr schön an den Verbformen aufzeigen, die den letztlich narrativen Grundzug dieser lyrischen LebensGeschichte untermauern. In der ersten Strophe das „me diste“, in der zweiten Strophe das „me siento“, in der dritten Strophe dann die Bewegung des Ich mit dem „me inclino“, danach die Vergangenheitsform des „Fuî“ und schließlich die Rückgabe des Gestundeten, des Geborgten, des Geschuldeten, in der Formel des „me entrego“. Stets ist das Personalpronomen des lyrischen Ich präsent und macht deutlich, dass es um die Geschichte des Lebens dieses Ichs in Form einer Rückschau auf eine ganze Karriere geht.

Die Stimmung eines Lebensendes stimuliert nur für den europäischen Leser eine überraschende Wendung im Schlusswort „mayo“, ist doch in unseren Breiten der Wonnemonat Mai nun gerade nicht das Zeichen des zu Ende gehenden Lebens. Juana de Ibarbourou aber lebt als Uruguayerin auf der Südhalbkugel; und so sind die Jahreszeiten außerhalb der Tropen in der gemäßigten Zone gänzlich anders semantisch fundiert. Im Mai treffen wir gleichsam mit dem ,November der Südhalbkugel` auf den Spätherbst des Lebens, steht doch der Winter nahe vor der Tür und zeigt das drohende Ende eines Zyklus an. Auch mit Blick auf diesen Lebenszyklus handelt es sich unstrittig um ein Abschiedsgedicht, das noch einmal die Seiten eines Dichterinnenlebens durchblättert. Soviel sei zu diesem geradezu klassischen Sonett aus dem letzten zu Lebzeiten veröffentlichten Gedichtband der Juana de Ibarbourou gesagt: Es ist ein Sonett, das etwas von der Ruhe und Heiterkeit jener Gedichte ausstrahlt, die einstmals den Ruhm der jungen Dichterin weit über die Grenzen Uruguays wie Lateinamerikas hinaus begründet hatten.

Es wäre in einem letzten Blick auf dieses Sonett überaus aufschlussreich, die Position Gottes mit jener des Vaters in Beziehung zu setzen, der nach späterem Bekunden Juanas für deren literarischen Weg durch sein ausgeprägtes Interesse an zeitgenössischer Lyrik mitverantwortlich war. Durch ihn empfing sie im Grunde das Wort, das lyrische Wort, das sie dann in ihren eigenen Schöpfungen wiedergab. Das Abschiedsgedicht an den männlichen Gott kann folglich ebenso als Abschiedsgedicht an den Vater gelesen werden, der Juana zur Literatur führte.

Bisweilen ist Juana de Ibarbourou wegen ihrer Herkunft aus gutem Hause ich hatte bereits erwähnt, dass sie mütterlicherseits der uruguayischen Oligarchie 
angehörte - angefeindet worden. Doch wir sollten aus ihr keine schlechtere Lyrikerin machen, bloß weil sie aus einem reichen und gebildeten Hause stammte, eine glückliche Kindheit genoss, eine vertrauensvolle Beziehung zu ihren Eltern und speziell zu ihrem Vater besaß und nicht jenes Leiden an ihrer Rolle und gesellschaftlichen Situation verkörperte, das man später geradezu von ihr verlangte! Nein, sie problematisierte ihre gesellschaftliche Herkunft nicht und machte sie auch nicht zum Ausgangspunkt ihres literarischen Schaffens.

Bei diesen gesellschaftlichen Voraussetzungen erscheint es als geradezu folgerichtig, dass Juana bereits 1914, also im Alter von neunzehn Jahren, den Bund der Ehe schloss und zwei Jahre später ihr einziges Kind gebar, natürlich einen Sohn mit dem nicht unprätentiösen Namen Julio César, so dass sich eine Lebensgeschichte abzeichnete, die keinesfalls die einer „poète maudite“ sein konnte und sein sollte. Doch wäre es ebenso unfair, Juana gegenüber Gabriela Mistral oder Alfonsina Storni abzuwerten, wie etwa einen César Vallejo auf Grund seiner einfachen Herkunft zuungunsten des reichen Vicente Huidobro aufzuwerten. Wir sollten uns stets auf die Lyrik konzentrieren, auch wenn die gesellschaftlichen Umstände den für unser Verständnis durchaus wichtigen Kontext zu diesen alles entscheidenden poetischen Texten bilden!

Hatten wir im Gedicht Soneto a Dios die Gottesliebe kennengelernt, so ist es nun die menschliche und die geschlechtliche Liebe, die wir bei der noch blutjungen Lyrikerin im Gedichtband Las lenguas de diamante von 1919 antreffen. Dabei möchte ich Ihnen gerne das Gedicht Fusión präsentieren, das keineswegs allein von einer Fusion der Seelen zu berichten weiß, sondern die Liebe in Bilder der Gewalt überträgt:

\footnotetext{
Meine Seele hat um Deine Seele einen Knoten gebildet, Dicht und dunkel.

Eine jede Umdrehung der übermenschlichen Bindung

Wird zur Wurzel, um sich tiefer zu verbinden.

Und sie ist eine unaufhörlich lange Umarmung,

Die nicht einmal der Tod zerstören wird. Fühlst Du nicht,

Wie ich mich selbst an Deinem Schatten nähre?

Meine Wurzel hat sich um Deine Wurzeln geflochten,

Und wenn Du den Knoten lösen wolltest,

Wirst Du es spüren, wie weh das tut im lebendigen Fleisch,

Und dass in meiner Verwundung Dein Blut noch strömt!

Und mit Deinen Händen wirst Du die Wunde heilen

Und fester, noch fester den Knoten ziehen! ${ }^{4}$
}

4 Ibarbourou, Juana de: Fusión. In (dies.): Obras completas, S. 33. 
Die Fusion zwischen dem lyrischen Ich und der zweiten Person Singular ist in diesem Gedicht zunächst auf Ebene der Seele angelegt - wie schon in der Rede von der „alma oscura“ im vorangehenden Gedicht. Doch im weiteren Verlauf dieses Gedichts zieht sich die Schlinge oder - um im Bild zu bleiben - der Knoten immer fester um die beiden Liebenden. Die Fusion dieses Liebespaares wird in Form der ersten beiden Verse bereits vorgeführt, insoweit hier die traditionelle Anordnung des Elfsilbers, der auch dieses Gedicht folgt, in den ersten beiden Versen sehr bewusst und gut sichtbar durchbrochen wird, so dass beide Verse ineinander übergehen oder, wie man auch sagen könnte, sich mit- und ineinander verknoten.

Die Verbindung der beiden Wesen wird dabei vom künstlichen, artifiziellen Bereich des Knotens in den naturbezogenen, organischen und vegetativen Bereich der Wurzeln und des physischen Verbunden-Seins übertragen, eine kleine, aber sehr bewusste Verschiebung, die durch eine nachfolgende weitere zum Körperlich-Fleischlichen zugleich auch die Dimensionen von Körper-Leib und Blut in diesem Gedicht zum Ausdruck bringt. Damit erfolgt eine Entwicklung vom Leblosen zum pflanzlich Lebendigen und von diesem $\mathrm{zu}$ einem lebendigen Organismus, der in der „carne viva“, der lebendigen Fleischlichkeit, seinen Höhepunkt erreicht.

In der Verbindung beider scheint zunächst eine Abhängigkeit des lyrischen Ich vom Anderen auf, insoweit sich die Seele des Ich um die Seele des Du herum lagert, sich windungsreich verknotet, um sich später selbst aus seinem Schatten zu ernähren. Es ist ein Vorgang, der geräuschlos abläuft, geradezu vegetativ angelegt ist, aber gegen Ende des Gedichts in den beiden Ausrufesätzen kulminiert, welche die ganze physische und seelische Gewalt dieses Prozesses, dieser Fusion im Sinne des Titels unseres Gedichts, repräsentieren.

Überaus aufschlussreich für unsere Deutung ist, dass eine ganze Reihe von Lexemen uns aus dem zuvor analysierten Soneto a Dios bekannt sind, darunter insbesondere „alma“ und „sombra“. Denn es ist im Schatten des Anderen, im Schatten des Du, wo das Ich seine eigene Lebensgrundlage unverrü ckbar schafft. Aber gerade in dieser Unverrückbarkeit, in dieser Unfähigkeit, herausgerissen, getrennt werden zu können, liegt wiederum die Bedrohung (und auch Drohung), die vom Ich für das ansonsten so aktive Du ausgeht. Die Fusion hat daher keineswegs nur anpassenden, anschmiegenden Charakter, sondern ist weit über jegliche Passivität hinaus verbunden mit dem Ende der Autonomie nicht nur des einen, sondern eben beider Körper. Diese hören gleichsam auf, separat noch lebensfähig zu sein. Die Fusion besitzt wie die Drohung einen übermenschlichen Charakter, insoweit der Knoten, die wechselseitige Durchdringung der beiden Wurzelstöcke, auch von Menschenhand nicht mehr aufzulösen ist. Denn der „lazo“, die Bindung und Verbindung beider Körper und Seelen, ist nicht von Menschen gemacht und 
so von Menschen auch nicht mehr zu lösen. Die Fundierung in abermals christlichen Metaphorologien und Vorstellungen, zugleich aber auch deren bewusste Transgredierung ist offenkundig.

Lassen Sie mich zum Abschluss noch ein drittes und letztes Gedicht aus dem Schaffen von Juana de Ibarbourou anführen! Ich wähle aus Zeitgründen nicht eines jener Gedichte, in denen die Bedingungen des Lebens als Frau reflektiert und bisweilen kontrastiv zu den Bewegungsfreiheiten der Männer in Szene gesetzt werden wie in dem durchaus gelungenen Gedicht Mujer, das vom sanften Feminismus seiner Autorin zeugt. Ich werde auf dieses Gedicht zu Beginn unserer Beschäftigung mit Alfonsina Storni zurückkommen. Da wir uns bei der argentinischen Lyrikerin noch ausführlich mit einer feministischen Ausrichtung beschäftigen werden und diese Dimension, so unterschiedlich sie auch formuliert sein mag, sicherlich auch das Gemeinsame des Postmodernismus Gabriela Mistrals, Juana de Ibarbourous und Alfonsina Stornis bildet, möchte ich jedoch an dieser Stelle darauf verzichten. Denn „Mujer“, also „Frau“, setzt seinen Auftaktvers „Si yo fuera hombre“ („Wenn ich ein Mann wär“) nicht in eine veränderte gesellschaftliche Position oder in eine konkrete, verwirklichbare Utopie mit transformierten geschlechterspezifischen Normen und Formen um, sondern stellt vielmehr die Unmöglichkeit der genderbezogenen Grenzüberschreitung dar, aller „ansias“, allem Begehren zum Trotz, welche das weibliche lyrische Ich empfindet.

Ich will Ihnen vielmehr das Titelgedicht jenes Bandes vorstellen, mit dem Juana de Ibarbourou an die Öffentlichkeit trat, um ebenso überraschend wie schlagartig berühmt zu werden. Es ist das Titelgedicht von Las lenguas de diamante, das noch in ihrem Soneto a Dios in dankbarer Erinnerung an den Beginn ihrer großen Karriere auftaucht:

Unter dem Vollmond, einer Oblate aus Kupfer gleich, Schweiften wir schweigend in vager Ekstase, Wie schmale Schatten, gleitend leicht über den Sand aus Bronce am Ufer des Sees.

Schweigen auf unseren Lippen eine Rose erblüht. Oh, wenn es meinen Geliebten zu sprechen gelüstet!, Die Blumenkrone würd' zerzaust wie ein verletzter Vogel Fallen und das sanfte Mysterium unterm Monde brechen.

Oh Götter, dass er nicht spräche! Mit der stärksten Binde, Die Ihr zur Hand habt, unterdrückt ihm die Stimm’! Und wenn nötig, so kratzt den steinernen Mantel des Todes, um die Binde seines Mundes zu bilden, hinein! 
Ich will nicht, dass er spricht. Ich will nicht, dass er spricht. Über dies erhabene Schweigen welch' Verwundung das Wort! Oh Zunge der Asche! Oh elende Zunge, versuch' es Nicht, dass das Sigel meiner Lippen jetzt bricht!

Unter dem Kupfer-Mond schweigsame Liebende Wir, mit den Augen stöhnend, mit den Augen sprechend. Unsere Pupillen sind gleich zwei Zungen von Diamanten, Bewegt von der Magie der höchsten Gespräche. ${ }^{5}$

In diesem Gedicht, das zugleich den gesamten Band Las lenguas de diamante und dessen ersten Teil, „La luz interior“, eröffnet, ist zweifellos die Dimension der Innerlichkeit, einer spannungsvoll gesteigerten Interiorität, mit dem Themenkomplex der Liebe oder, vorsichtiger ausgedrückt, der Präsenz des geliebten Anderen verknüpft. Wieder ist es eine Zweisamkeit zwischen dem lyrischen Ich und einem wohl eher männlichen Partner, der aber geschlechtlich nicht determiniert ist. Dem Gedicht selbst, im Titel angelegt, liegt die Bewegung einer Entkörperlichung, einer Bewegung vom direkten Kontakt - etwa über die menschliche Sprache, aber auch die menschliche Zunge - hin zu einem in vielerlei Hinsicht unorganischen Kontakt, dem ,Diamantinen‘ der Augen, zu Grunde. Denn die Ekstase der beiden Liebenden vollzieht sich in der geräuschlosen Bewegung als schweigsame Schatten, die unter der Kupferscheibe des Mondes am Ufer eines Sees lustwandeln. Das Organ der Zunge und damit das Sprechen, das körperliche Verlauten, müssen dem Augenkontakt, dem Gespräch zweier Augenpaare, und damit dem Fern-Sinn des menschlichen Auges weichen, das überdies noch in Diamanten verwandelt wird. Alles Körperliche ist ausgeschlossen.

Auf Ebene der Wortwahl steht dieses in Vierzehnsilbern gehaltene Gedicht unverkennbar in der Tradition des Modernismo, was auch schon die Farbwerte und das Dekor der ersten Strophe anzeigen. Insbesondere die „arenas de bronce“ und die Situierung an den Ufern eines Sees weisen deutlich auf die modernistische Provenienz dieses Textes hin, der sich einer Sprache bedient, die Rubén Darío nicht allzu ferne zu stehen scheint. Was dieses Gedicht freilich stark von dessen Modell unterscheidet, ist die völlig zurückgenommene Körperlichkeit, die beim nikaraguanischen Dichter stets in starker Plastizität vorhanden ist.

Die erste Strophe ist eine Zustandsbeschreibung, die auf der Vereinigung zu einer ersten Person Plural beruht und im Zeichen einer vagen Ekstase steht. Selbst in dieser ersten Strophe ist bereits eine Entkörperlichung feststellbar, sind die Gestalten der beiden Liebenden doch inszeniert als schlanke Schatten, die sich im

5 Ibarbourou, Juana de: Las lenguas de diamante. In (dies.): Obras completas, S. $97 \mathrm{f}$. 
Lichte des Vollmonds geräuschlos bewegen. Das Licht, so können wir anmerken, scheint in dieser Szenerie nicht so sehr von innen zu kommen, sondern eben von jener hellen Scheibe, in deren Zeichen das Gedicht steht und die am Ende wieder aufgenommen werden wird. Doch in diesem kalten Mondlicht zeigt sich das „Wir“ nur als Schatten, fast gespensterhaft.

Der ersten von fünf aus jeweils vier Versen von Vierzehnsilbern bestehenden Strophen des Gedichts, die syntaktisch als eine Einheit, als einzelne Sätze, ausgeführt sind, steht gleich im ersten Vers der zweiten Strophe eine abgeschlossene Satzperiode gegenüber. Diese weist eine gewisse Agrammatizität auf, insoweit „en nuestros labios“ sich sowohl auf das „silencio“ als auch auf die nachfolgende Rose beziehen lässt und einen für das Gedicht charakteristischen fließenden Übergang vorführt. Die Rose steht selbstverständlich für die Liebe der beiden ekstatisch Entrückten, ihr Aufblühen repräsentiert den „moment d'amour“. Die Lippen der Liebenden scheinen etwas geöffnet, insofern wir dort eine Rose aufblühen sehen, sind aber noch nicht - wie im weiteren Verlauf des Gedichts - aufeinander gepresst, um keinen Laut nach außen tragen zu können und zugleich, um jeglichen Zugang zu einem Innenraum zu verwehren. Noch wird ihnen die Binde nicht aufgedrückt, welche den Mund verschließen und die körperliche, fleischliche Zunge gleichsam wegschließen soll. Alles Fleischliche, das zu Asche zerfällt, wird aus dem Gedicht verbannt.

Das Schweigen, das semantisch bereits explizit in der ersten Strophe durch „taciturnos“ eingeführt und in der zweiten Strophe von Beginn an aufgenommen wird, erscheint als ungebrochen, obwohl es als ein Dialog der beiden Liebenden im höchsten Maße beredt ist. Es ruht auf den Lippen der gleichsam schweigend Sprechenden, wobei freilich nicht beide freiwillig schweigen. Die topische Metapher der Lippen als Rose wird dabei insoweit variiert, als nun auf den Lippen eine Rose aufblüht, ein organischer, vegetativer Vorgang, der Zugleich eine Einheit - die Einheit einer einzigen Rose auf den Lippen der Liebenden - und die Unmöglichkeit des Sprechens zum Ausdruck bringt. Doch die Rose auf den Lippen garantiert in den Augen des lyrischen Ich noch nicht das nachhaltige ekstatische Schweigen auch des oder der Anderen. Besteht nicht die Gefahr, dass dieses Du das Schweigen bricht?

Gleichzeitig wird das für das Ich so bedrohliche Akustische in ein Visuelles umgewandelt, wird die Kommunikation durch Sprache und Sprechen durch eine optische Kommunikation ersetzt - prekär, wie sich zeigen wird. Zugleich erscheint aber auch eine Spaltung des „Wir“ in der Wendung „mi amante“, in welcher erste und dritte Person auseinandertreten, wobei die erste Person als Possessivum „mi“ eingeführt und auf den Liebenden oder die Liebende - denn ,amante“ wird nicht geschlechtlich determiniert - bezogen wird. Die Nicht-Determinierung des Geschlechts hat zweifellos etwas mit der Ausschließung des Körperlich-Leibhafti- 
gen wie des Fleischlichen zu tun, so dass die schlanken Schatten der schweigend Umherschweifenden gänzlich vom Körperlichen ,abgezogen' werden und damit abstrakt bleiben. Es geht in keiner Weise wie im Gedicht Fusión um eine körperliche Fusion oder Vereinigung.

Die Versuchungen, denen der oder die „amante“ ausgesetzt ist, betreffen das Sprechen, das In-den-Mund-Nehmen, ein in Worte fassen, das paradoxerweise für das in Worte gefasste Gedicht zur Bedrohung eben jener vagen Ekstase der ersten Strophe wird, welche in der zweiten Strophe als „suave misterio sublunar“ in anderer Wendung erscheint. Zweifellos ist in diesen Versen die Vereinigung der Liebenden in einer mystischen irdischen Liebe („misterio“ und „sublunar“) gefährdet, also gleichsam in jener „unio mystica“, in welcher sich in unkörperlicher Weise die Seele des Mystikers oder der Mystikerin mit Gott verbindet. Und diese Gefährdung geht aus von einem profanen Sprechen. Die Blumenkrone, die die Vermählung der Braut anzeigt, würde $\mathrm{zu}$ Boden fallen wie ein verwundeter Vogel, auch dies ein topischer Vergleich, der durchaus in romantischer Tradition steht, die Dimension des Mystischen aber durch die Präsenz der Braut aufrecht erhält. Es besteht kein Zweifel, dass Juana de Ibarbourou in ihrem Gedicht auf die Magie mystischer Kommunikation und damit auf eine Seelenvereinigung anspielt.

Vor diesem Hintergrund mystischer Liebe ist es nicht verwunderlich, dass sich das lyrische Ich an die Götter wendet, wenn auch der Plural gleichsam historisch über das Christentum hinausschießt. So wird jene abendländische Antike eingeblendet, welche die bevorzugte Projektionsfläche der hispanoamerikanischen Modernisten war, wie wir in vielen Gedichten, nicht zuletzt aber auch in Rubén Daríos berühmtem erotischen Liebesgedicht Leda sehen können. Die Anrufung der Götter ist freilich zugleich Aufruf zur Tat, zum Eingreifen: Sie sollen die Stimme des Du, die zur Artikulation drängt, unterdrücken, ersticken, sozusagen unter-binden, ein Akt der Gewalt und Grausamkeit, der schieren Unterdrückung und Repression, der bereits im vorigen Gedicht sinnlich präsent war und die Liebeslyrik der Ibarbourou offenkundig prägt. Denn diese Liebeslyrik erscheint nur auf den ersten Blick als sanft, gibt sich bei genauerer Analyse jedoch als gewalttätig zu erkennen.

Die Götter treten im Gedicht gleichsam als dritte, angerufene und herbeigerufene Partei hinzu, nicht aber - wie man vermuten könnte - als Vermittler, sondern in einer vom Subjekt gewünschten, erhofften und befohlenen unterdrückenden Rolle. Die Anleitung zu diesem göttlichen Tun bildet der zweimal wortgleich wiederholte Befehl des ersten Verses der vierten Strophe: „Yo no quiero que hable. Yo no quiero que hable.“ Die Wiederholung dieser Willensbekundung macht den Absolutheitsanspruch des lyrischen Ich deutlich, das Schweigen nicht durch das Wort, nicht durch den persönlichen Akzent brechen zu lassen. 
Das Wort wäre eine gleichsam körperliche Beziehung zwischen den Liebenden: Es würde die Situation mystischer Ekstase, mystischer Vereinigung zerstören. Daher die Abwertung nicht nur des Wortes - gleichsam akustisches Äquivalent des Körperlichen, der Resonanz im Körper-Leib -, sondern vor allem auch der „lengua de ceniza“, der „lengua miserable“, welche als Repräsentation des Fleischlichen in diesen Wendungen in ihrer Vergänglichkeit und Erbärmlichkeit aufgerufen wird. Denn die fleischliche Zunge steht ganz dem entrückten Zustand der irdischen, sublunaren Welt der Ekstase im Wege. Zweifellos ist das Lexem „la lengua“ in seiner Doppelbedeutung ebenso die Sprache, der das Wort angehört also gleichsam im Saussure’schen Sinne die Unterscheidung zwischen „langue“ und „parole“, zwischen dem System menschlicher Sprache und deren konkreter, individueller Realisierung oder Sprachäußerung. Ebenso ist sie jenes Organ, das die geschlossenen Lippen durchdringen kann und fleischlich in den anderen Mund, in den Mund und Körper des Anderen eindringt. Eben dies aber soll mit göttlicher Hilfe und Gewalt verhindert, unterbunden, unterdrückt werden.

Die fünfte und letzte Strophe stellt in der Wiederaufnahme und Variation bestimmter Lexeme der ersten Strophe eine Replik oder ein Wiederanknüpfen an den ekstatischen Zustand der ersten Strophe her, wobei das lyrische Ich an den Anderen oder die Andere appelliert, zum Zustand der „taciturnos amantes“ zurückzukehren. Noch ist kein Wort gefallen, wurde das Schweigen nicht durchbrochen, ja gab es nicht einmal einen erkennbaren Versuch dies zu tun. Und doch ist das lyrische Ich nicht nur alarmiert, sondern ergreift Gegenmaßnahmen gegen ein vermeintliches Durchbrechen des Schweigens. Die Anrufung der Götter geht einher mit einer Anrufung höherer Gewalt, welche auf die oder den „amante“ ausgeübt werden soll. Die von den Göttern erbetene und zumindest virtuell ausgeübte Gewalt kann man getrost als brutal umschreiben.

Das Instrument der schweigsamen Kommunikation sollen künftig allein die Augen sein, „los ojos“, die gleich zweimal als Medium des Gesprächs oder Dialogs angerufen werden. Damit ist von allen Sinnen, von allen Formen sinnlicher Wahrnehmung der unkörperlichste Sinn favorisiert, der Fernsinn des Gesichtssinnes, der in der Tat auch in der Tradition der Mystik eine besondere Rolle einnimmt. Im Gegensatz zur dominanten katholischen Hierarchie der Sinne, in welcher das Ohr als das eigentliche Organ des Christenmenschen erscheint und alles auf das GeHorchen des Christen ausgerichtet ist, wird wie bei den spanischen Mystikerinnen und Mystikern der Gesichtssinn bevorzugt als Sinn der Erhebung, des spirituellen „recogimiento“, der inneren Sammlung. Las lenguas de diamante knüpft in diesem Bereich an eine lange ebenso literarische wie mystische Traditionslinie an.

Denn das Bild, auch das spirituelle Bild, dringt in uns ein, gerade auch das Bild des Leidens, wie es Santa Teresa de Jesús in ihren Anleitungen zum mystischen Schauen Gottes betonte. Kein profanes gesprochenes Wort sollte dieses 
mystische Schauen beeinträchtigen. Also kein Sprechen und Hören, kein - um bei der Zunge zu bleiben - Schmecken, auch kein Tasten mit den Händen, kein Riechen: All die anderen, körperlichen und auf körperlichem Kontakt beruhenden Sinne sind aus dieser ekstatischen Schau ausgeschlossen. Allein der am stärksten vergeistigte Sinn wird angerufen. Allein auf diese Weise verwandeln sich die Pupillen in Medien von „diálogos supremos“, wird aus der Zungensprache eine diamantene Augensprache, die allein fähig ist, „,sublunar“ das Sublime auszudrücken, den rational nicht fassbaren Teil menschlicher Kommunikation. Das ekstatische Erleben der beiden Liebenden wird dergestalt zu einer körperfernen und doch gemeinsamen visuellen Schau, die gleichzeitig im Zeichen des Schmuckes, des Juwels, der anorganischen Diamanten steht.

Dieses Gedicht aus dem Schweigen, dieses Gedicht einer damals noch unbekannten Dichterin, mag vielleicht auch darum einen so großen und sofortigen Erfolg erzielt haben, weil es nicht allein dieses Schweigen evoziert und zugleich durchbricht, sondern dies auch in erreichbarer, literarisch durchaus nicht revolutionärer Form tut. Vielmehr entwickelte es seine eigene Sprache in Anknüpfung an modernistische Positionen, wobei es diese aber zugleich auf - zumindest von Rubén Darío her gesehen - einfachere Formeln zurückführt. Das Gedicht öffnet sich sehr weit auf eine Leserschaft hin, deren Aneignung des Gedichts sich vor dem Hintergrund eines modernistisch geprägten Lektürehorizonts vollzieht. Weder Lexik noch Chromatik stellten besondere Anforderungen an diese mit dem Modernismo vertraute Leserschaft; allein die Unkörperlichkeit, die Stimme einer Dichterin mit dem mit ihr verbundenen weiblichen Ich sowie die geschlechtliche Undeterminiertheit der Liebenden konnten zusammen mit der Gewalt, die sich in diesen Versen ausspricht, noch erstaunen.

Nichts oder nur wenig ist hier mehr von jener lasziven, erotisch aufgeladenen, etwas morbiden Atmosphäre zu spüren, wie wir sie bei vielen modernistischen Dichtern (vielleicht mit Ausnahme José Martís) beobachten können. Zugleich bewegt sich die Bildersprache durchaus noch in modernistischen Bahnen, so dass wir auch dieses Gedicht sehr wohl einer spätmodernistischen lyrischen Schreibweise zuordnen können, die sich - wie wir sahen - mit Federico de Onís als „Postmodernismo“ bezeichnen ließe.

Parallel hierzu verweist die mystische Grundhaltung auch auf eine Tendenz hin zum Göttlichen, zum Transzendenten, zum Übermenschlichen: Die Problematik der spanischen Mystik war den hispanoamerikanischen Modernisten durchaus nicht fremd, sondern sehr vertraut. Denn die poetischen Werke der Mystikerinnen und Mystiker des 16. Jahrhunderts waren ein wesentlicher und selbstverständlicher Bestandteil der Lektüre von Autoren wie José Martí, Rubén Darío, Julián del Casal oder José Enrique Rodó, der in seinem Ariel von 1900 wiederholt auf Topoi der spanischen Mystik zurückgriff. 
Und doch findet sich bei Juana de Ibarbourou, wohl am nächsten noch zu José Martí, jene Spiritualität, die sich nicht nur den ästhetischen Überschuss dienstbar macht, sondern versucht, zu den letzten Dingen in einer Art „unio mystica“ vorzudringen. Dass dies eine Frau tat, musste von den Zeitgenossen nicht als revolutionär empfunden werden, sondern verstärkte gerade eine Deutung, welche die Liebe vom Körperlichen abzulösen und $\mathrm{zu}$ abstrahieren suchte, um in eben dieser Ablösung und Abstraktion die Vereinigung, die mystische Ekstase zu finden. Dass Juana de Ibarbourou - wie wir schon im Gedicht Fusión sahen - aber auch die Körpermetaphern stark zu machen und bis in Gewaltszenen hinein auszudehnen wusste, konnten wir auch in Las lenguas de diamante mühelos begreifen.

An dieser Stelle ließe sich ein Interpretationsansatz einfügen, auf den ich noch einmal im Zusammenhang mit Alfonsina Storni zurückkommen will. Er geht aus von der Inszenierung von Körperlichkeit im gesamten Band Las lenguas de diamante, aber auch in seinem Titelgedicht, wo sich eine bemerkenswerte Inszenierung von Lippen der Leserin und dem Leser förmlich aufdrängt, jener Lippen also, die besonders in der vierten Strophe eine so wichtige Rolle übernehmen. An dieser Stelle sind es die Lippen, die durch die Zunge gleichsam geöffnet werden. Erblicken wir im Liebenden ein männliches Subjekt, welches die Stille und damit auch die „unio mystica“ der beiden Liebenden bedroht, dann entsteht diese Vereinigung des weiblichen Subjekts nur vor dem Hintergrund des Schweigens des männlichen „amante“, der als ,stummer‘ Gesprächspartners scheinbar in den Hintergrund tritt.

Die Lippen der Liebenden bleiben geschlossen, wobei sie - erinnern wir uns an den ersten Vers der zweiten Strophe - mit einer Blume, der Rose, besetzt und assoziiert werden. Diese Blume der Liebe wäre auch mit einem Ort der Liebe in Verbindung zu bringen, dessen Lippen im Sinne der Entkörperlichung ebenfalls geschlossen bleiben. Nichts vermag sie zu öffnen, nichts dringt durch sie hindurch. An dieser Stelle ließe sich wiederum ein Interpretationsansatz einbringen, der auf die Theorie der feministischen Theoretikerin Luce Irigaray zurückgreift, der zufolge weibliche Lust gerade durch die männliche Penetration der weiblichen Lippen zerstört werde. Diese Lust entstehe gerade durch das AneinanderReiben der Lippen, durch eine eigene Form von Geschlechtlichkeit, in welcher die Präsenz des Mannes nur zerstörend wirken kann.

Vor diesem Hintergrund wirkt der zweifach ausgesprochene Befehl „Ich will nicht, dass er spricht“, wie ein Verbot, den Ort der Rose zu durchdringen und mit der „Zunge der Asche“ zu berühren. Dieser auf Luce Irigaray zurückgehende Ansatz könnte auf diese Fragestellung fruchtbar angewandt werden, Licht in diese nur dunkel beleuchtete Passage bringen und sich auch noch mit der nachfolgenden Interpretation des Gedichts Mujer von Juana de Ibarbourou verbinden 
lassen. Doch wir wollen derartige Fragestellungen und Deutungsansätze besser im nachfolgenden Kapitel behandeln, das nach der großen chilenischen und der großen uruguayischen Dichterin nun der nicht minder großen argentinischen Lyrikerin dieses Dreigestirns gewidmet ist. 